\title{
EXISTENCE AND UNIQUENESS OF SOLUTIONS FOR A SEMILINEAR ELLIPTIC SYSTEM
}

ROBERT DALMASSO

Received 23 February 2005 and in revised form 4 May 2005

We consider the existence, the nonexistence, and the uniqueness of solutions of some special systems of nonlinear elliptic equations with boundary conditions. In a particular case, the system reduces to the homogeneous Dirichlet problem for the biharmonic equation $\Delta^{2} u=|u|^{p}$ in a ball with $p>0$.

\section{Introduction}

In this paper, we are interested in the existence, the nonexistence, and the uniqueness question for the following problem:

$$
\begin{gathered}
\Delta u=|v|^{q-1} v \quad \text { in } B_{R} \\
\Delta v=|u|^{p} \quad \text { in } B_{R} \\
u=\frac{\partial u}{\partial v}=0 \quad \text { on } \partial B_{R}
\end{gathered}
$$

where $B_{R}$ denotes the open ball of radius $R$ centered at the origin in $\mathbb{R}^{n}(n \geq 1), \partial / \partial v$ is the outward normal derivative, and $p, q>0$.

Concerning uniqueness, we have the following theorem.

Theorem 1.1. (i) Let $p>0, q \geq 1$ with $p q \neq 1$. Then (1.1) has at most one nontrivial radial solution $(u, v) \in\left(C^{2}\left(\bar{B}_{R}\right)\right)^{2}$.

(ii) Let $p>0, q \geq 1$ with $p q=1$. Assume that (1.1) has a nontrivial radial solution $(u, v) \in\left(C^{2}\left(\bar{B}_{R}\right)\right)^{2}$. Then all nontrivial radial solutions are given by $\left(\theta^{q} u, \theta v\right)$, where $\theta>0$ is an arbitrary constant.

When $q=1$ and $p \in(0,1) \cup(1, \infty)$, Theorem 1.1 was established in [4] (see also the references therein). When $n=1, q=1$, and $p>1$, the uniqueness of a nontrivial solution follows from a general result given in [5].

When $q=1, p>1$, and

$$
p<\frac{n+4}{n-4} \quad \text { if } n \geq 5
$$


the existence of a nontrivial solution was proved in $[2,5,11]$. The case $q=1$ and $0<p<1$ is well known: see, for instance, $[4,6]$. Moreover, when $q=1$, any nontrivial solution of (1.1) is positive in $B_{R}$ because the Green function of $\Delta^{2}$ with Dirichlet boundary conditions is positive in $B_{R}[1,8]$. Then it was proved in $[2,11,12]$ that problem $(1.1)$ has no nontrivial solutions, whether radial or not, if

$$
p \geq \frac{n+4}{n-4} \quad(n \geq 5)
$$

We will prove a nonexistence result and an existence result.

Theorem 1.2. Suppose $n \geq 3$. Let $p, q>0$ satisfy

$$
\frac{1}{p+1}+\frac{1}{q+1} \leq \frac{n-2}{n}
$$

(i) Let $(u, v) \in\left(C^{2}\left(\bar{B}_{R}\right)\right)^{2}$ be a solution of problem (1.1) such that $u \geq 0$ in $B_{R}$. Then $u=v=0$.

(ii) If $(u, v) \in\left(C^{2}\left(\bar{B}_{R}\right)\right)^{2}$ is a radial solution of problem (1.1), then $u=v=0$.

Theorem 1.3. (i) Let $p>0, q \geq 1$ with $p q \neq 1$ satisfy

$$
\frac{1}{p+1}+\frac{1}{q+1}>\frac{n-2}{n} \quad \text { if } n \geq 3 \text {. }
$$

Then (1.1) has a nontrivial radial solution $(u, v) \in\left(C^{2}\left(\bar{B}_{R}\right)\right)^{2}$.

(ii) Let $p>0, q \geq 1$ with $p q=1$. Then there exists $R>0$ such that (1.1) has a nontrivial radial solution $(u, v) \in\left(C^{2}\left(\bar{B}_{R}\right)\right)^{2}$.

Remark 1.4. Notice that when $p q \leq 1$, (1.5) holds.

In the sequel, $\Delta$ denotes equally the Cartesian and the polar form of the Laplacian.

In Section 2, we give some preliminary results. Theorem 1.1 is proved in Section 3 using the same approach as in [4, 7]. In Section 4, we prove Theorem 1.2. We prove Theorem 1.3 in Section 5: the proof is based on a two-dimensional shooting argument for the ordinary differential equations associated to radial solutions of $(1.1)[3,5,7,15,16]$. The fact that $q \geq 1$ is crucial in the proofs of Theorems 1.1 and 1.2.

\section{Preliminaries}

In this section, we first examine the structure of nontrivial radial solutions of (1.1).

Lemma 2.1. Let $(u, v) \in\left(C^{2}\left(\bar{B}_{R}\right)\right)^{2}$ be a nontrivial radial solution of $(1.1)$. Then $u^{\prime}<0$ on $(0, R), \Delta u(R)=u^{\prime \prime}(R)>0$ and $v^{\prime}>0$ on $(0, R], v(0)<0<v(R)$.

Proof. Clearly $u=0$ if and only if $v=0$. We have

$$
r^{n-1} v^{\prime}(r)=\int_{0}^{r} s^{n-1}|u(s)|^{p} d s \geq 0, \quad 0 \leq r \leq R
$$

Assume that $v(0) \geq 0$. Then (2.1) implies that $v \geq 0$ on $[0, R]$, hence $\Delta u \geq 0$ on $[0, R]$. Therefore $r^{n-1} u^{\prime}(r)$ is nondecreasing in $[0, R]$. Since $u^{\prime}(0)=u^{\prime}(R)=u(R)=0$, we deduce 
that $u=0$ and we reach a contradiction. The case where $v(R) \leq 0$ can be handled in the same way. Therefore we have $v(0)<0<v(R)$. We claim that $u(0) \neq 0$. Indeed assume that $u(0)=0$. Using (2.1) and the first equation in (1.1), we deduce that there exists $R^{\prime} \in$ $(0, R)$ such that $r^{n-1} u^{\prime}(r)$ is nonincreasing in $\left[0, R^{\prime}\right]$ and nondecreasing in $\left[R^{\prime}, R\right]$. Since $u^{\prime}(0)=u^{\prime}(R)=0$, we obtain that $u^{\prime} \leq 0$ in $[0, R]$. Using the fact that $u(0)=u(R)=0$, we deduce that $u=0$ in $[0, R]$ and we get a contradiction. Now (2.1) implies that $v^{\prime}>0$ in $(0, R]$. Let $R^{\prime} \in(0, R)$ be such that $v\left(R^{\prime}\right)=0$. Using the first equation in (1.1), we deduce that $r^{n-1} u^{\prime}(r)$ is decreasing in $\left[0, R^{\prime}\right]$ and increasing in $\left[R^{\prime}, R\right]$. Since $u^{\prime}(0)=u^{\prime}(R)=0$, we obtain $u^{\prime}<0$ in $(0, R)$.

Lemma 2.2. Assume that $n \geq 1$ and $p, q>0$. Let $\alpha, \beta>0$ be fixed. If $(u, v) \in\left(C^{2}\left(\mathbb{R}^{n}\right)\right)^{2}$ is a radial solution of

$$
\begin{gathered}
\Delta u=|v|^{q-1} v, \quad r>0, \\
\Delta v=|u|^{p}, \quad r>0, \\
u(0)=\alpha, \quad u^{\prime}(0)=v^{\prime}(0)=0
\end{gathered}
$$

such that $u u^{\prime}<0$ on $(0, \infty)$, then $v<0$ on $(0, \infty)$.

Proof. We have $0<u \leq \alpha$ on $[0, \infty)$. Therefore

$$
r^{n-1} v^{\prime}(r)=\int_{0}^{r} s^{n-1} u(s)^{p} d s>0 \quad \text { for } r>0 .
$$

Assume that the conclusion of the lemma is false. Then (2.3) implies that there exist $a, b>0$ such that

$$
v(r) \geq a \quad \text { for } r \geq b .
$$

We deduce that

$$
\left(r^{n-1} u^{\prime}(r)\right)^{\prime} \geq a^{q} r^{n-1} \quad \text { for } r \geq b,
$$

hence

$$
r^{n-1} u^{\prime}(r) \geq a^{q} \frac{r^{n}-b^{n}}{n}+b^{n-1} u^{\prime}(b) \quad \text { for } r \geq b
$$

which implies that $u^{\prime}(r)>0$ for $r$ large and we reach a contradiction.

Now we give a lemma which is needed in the proof of Theorem 1.3.

Lemma 2.3. Assume that $n \geq 1$ and $p, q>0$. Let $\alpha, \beta>0$ be fixed. Assume that for some $a>0,(u, v) \in\left(C^{2}\left(\bar{B}_{a}\right)\right)^{2}$ is a radial solution of

$$
\begin{gathered}
\Delta u=|v|^{q-1} v \quad \text { in }[0, a], \\
\Delta v=|u|^{p} \quad \text { in }[0, a], \\
u(0)=\alpha, \quad u^{\prime}(0)=v^{\prime}(0)=0
\end{gathered}
$$


1510 Existence and uniqueness for an elliptic system

such that $u u^{\prime}<0$ on $(0, a)$. Then

$$
|v(r)| \leq d \max \left(\beta, \alpha^{(p+1) /(q+1)}\right), \quad 0 \leq r \leq a,
$$

where

$$
d=\left(1+\frac{q+1}{p+1}\right)^{1 /(q+1)} .
$$

Proof. We have $0<u \leq \alpha$ on $[0, a)$. As in Lemma 2.2 we deduce that $v^{\prime}>0$ on $(0, a]$. We have

$$
\int_{0}^{r}\left(v^{\prime} \Delta u+u^{\prime} \Delta v\right) d s=\int_{0}^{r}\left(|v|^{q-1} v v^{\prime}+u^{p} u^{\prime}\right) d s
$$

for $r \in[0, a]$. Since

$$
\begin{aligned}
\int_{0}^{r}\left(v^{\prime} \Delta u+u^{\prime} \Delta v\right) d s & =\int_{0}^{r}\left(u^{\prime} v^{\prime}\right)^{\prime} d s+2(n-1) \int_{0}^{r} \frac{u^{\prime}(s) v^{\prime}(s)}{s} d s \\
& =u^{\prime}(r) v^{\prime}(r)+2(n-1) \int_{0}^{r} \frac{u^{\prime}(s) v^{\prime}(s)}{s} d s, \\
\int_{0}^{r}\left(|v|^{q-1} v v^{\prime}+u^{p} u^{\prime}\right) d s & =\frac{|v(r)|^{q+1}}{q+1}+\frac{u(r))^{p+1}}{p+1}-\frac{\beta^{q+1}}{q+1}-\frac{\alpha^{p+1}}{p+1},
\end{aligned}
$$

we obtain

$$
\frac{|v(r)|^{q+1}}{q+1}+\frac{u(r))^{p+1}}{p+1}=\frac{\beta^{q+1}}{q+1}+\frac{\alpha^{p+1}}{p+1}+u^{\prime}(r) v^{\prime}(r)+2(n-1) \int_{0}^{r} \frac{u^{\prime}(s) v^{\prime}(s)}{s} d s
$$

for $r \in[0, a]$, which implies that

$$
|v(r)|^{q+1} \leq \beta^{q+1}+\frac{q+1}{p+1} \alpha^{p+1}, \quad 0 \leq r \leq a,
$$

and the lemma follows.

\section{Proof of Theorem 1.1}

(i) Let $(u, v)$ and $(w, z)$ be two nontrivial radial solutions of (1.1). Let $s$ and $t$ be defined by

$$
s=2 \frac{q+1}{p q-1}, \quad t=2 \frac{p+1}{p q-1} .
$$

For $\lambda>0$ we set

$$
\widetilde{w}(r)=\lambda^{s} w(\lambda r), \quad \widetilde{z}(r)=\lambda^{t} z(\lambda r), \quad 0 \leq r \leq \frac{R}{\lambda}
$$


By Lemma 2.1, $\widetilde{w}>0$ on $[0, R / \lambda)$ and then we have

$$
\begin{gathered}
\Delta \widetilde{w}(r)=|\widetilde{z}(r)|^{q-1} z(r), \quad 0 \leq r \leq \frac{R}{\lambda}, \\
\Delta \widetilde{z}(r)=\widetilde{w}(r)^{p}, \quad 0 \leq r \leq \frac{R}{\lambda}, \\
\widetilde{w}\left(\frac{R}{\lambda}\right)=\widetilde{w}^{\prime}\left(\frac{R}{\lambda}\right)=0 .
\end{gathered}
$$

Choose $\lambda$ such that $\lambda^{s} w(0)=u(0)$. Then we have

$$
\widetilde{w}(0)=u(0)
$$

We want to show that

$$
\widetilde{z}(0)=v(0)
$$

Suppose that $\tilde{z}(0)<v(0)$. If there exists $a \in(0, \min (R, R / \lambda)]$ such that $\tilde{z}-v<0$ on $[0, a)$ and $(\tilde{z}-v)(a)=0$, then $\Delta(\widetilde{w}-u)<0$ on $[0, a)$. Equation (3.4) and the maximum principle imply that $\tilde{w}-u<0$ on $(0, a]$. Therefore $\Delta(\tilde{z}-v)<0$ on $(0, a]$ and the maximum principle implies that $\tilde{z}-v>(\tilde{z}-v)(a)=0$ on $[0, a)$, a contradiction. Thus $\tilde{z}-v<0$ on $[0, \min (R, R / \lambda)]$. Then, as before, we show that $\widetilde{w}-u<0$ on $(0, \min (R, R / \lambda)]$. Since

$$
(\tilde{w}-u)\left(\min \left(R, \frac{R}{\lambda}\right)\right)= \begin{cases}-u\left(\frac{R}{\lambda}\right) & \text { if } \lambda>1, \\ 0 & \text { if } \lambda=1, \\ \tilde{w}(R) & \text { if } \lambda<1,\end{cases}
$$

we deduce that $\lambda>1$ with the help of Lemma 2.1. Now using the fact that $r^{n-1}(\widetilde{w}-u)^{\prime}(r)$ is decreasing in $[0, R / \lambda]$, we get $(\widetilde{w}-u)^{\prime}(R / \lambda)<0$. Since $(\widetilde{w}-u)^{\prime}(R / \lambda)=-u^{\prime}(R / \lambda)>0$ by Lemma 2.1, we again obtain a contradiction. The case $\tilde{z}(0)>v(0)$ can be handled in the same way. Thus (3.5) is proved.

Now we define the functions $U, W, F$, and $G_{n}$ by

$$
\begin{array}{cc}
U(r)=(u(r), v(r)), & 0 \leq r \leq R, \\
W(r)=(\tilde{w}(r), \tilde{z}(r)), & 0 \leq r \leq \frac{R}{\lambda}, \\
F(x, y)=\left(|y|^{q-1} y, x^{p}\right), & x \geq 0, y \in \mathbb{R}, \\
G_{n}(r, s)=\left\{\begin{array}{lc}
r-s & \text { if } n=1, \\
s \ln \left(\frac{r}{s}\right) & \text { if } n=2, \\
\frac{s}{n-2}\left(1-\left(\frac{s}{r}\right)^{n-2}\right) & \text { if } n \geq 3
\end{array}\right.
\end{array}
$$


1512 Existence and uniqueness for an elliptic system

for $0 \leq s \leq r$. Using (3.4), (3.5), and the fact that $u^{\prime}(0)=\widetilde{w}^{\prime}(0)=v^{\prime}(0)=\widetilde{z}^{\prime}(0)=0$, we easily obtain

$$
U(r)-W(r)=\int_{0}^{r} G_{n}(r, s)(F(U(s))-F(W(s))) d s
$$

for $r \in[0, \min (R, R / \lambda)]$. When $p \geq 1, F$ is locally Lipschitz continuous, and using Gronwall's lemma we obtain $U=W$ on $[0, \min (R, R / \lambda)]$. When $p \in(0,1)$, let $a \in(0, \min (R$, $R / \lambda)$ ) be fixed. Then $u(0) \geq u(r) \geq u(a)>0, \widetilde{w}(0)=u(0) \geq \widetilde{w}(r) \geq \widetilde{w}(a)>0$ for $r \in[0, a]$. Since $F$ is locally Lipschitz continuous on $(0,+\infty) \times \mathbb{R}$, as before we obtain $U=W$ on $[0, a]$. By continuity we get $U=W$ on $[0, \min (R, R / \lambda)]$. Now we deduce that $\lambda=1$ and thus $(u, v)=(w, z)$ on $[0, R]$.

(ii) Let $(u, v)$ be a nontrivial radial solution of problem (1.1). Then, for any $\theta>0$, $(w, z)=\left(\theta^{q} u, \theta v\right)$ is a nontrivial radial solution of problem (1.1). Now let $(w, z)$ be a nontrivial radial solution of (1.1). Choose $\theta>0$ such that $\theta^{q} u(0)=w(0)$ and define $\widetilde{w}=\theta^{q} u$, $\tilde{z}=\theta v$. Then $(\widetilde{w}, \widetilde{z})$ is a nontrivial radial solution of $(1.1)$ such that $\widetilde{w}(0)=w(0)$. Arguing as in part (i), we show that $\tilde{z}(0)=z(0)$ and that $(\tilde{w}, \tilde{z})=(w, z)$.

Remark 3.1. Our technique also applies when there is a homogeneous dependence on the radius $|x|$. More precisely, for $p>0, q \geq 1$, and $p q \neq 1$, the following system

$$
\begin{gathered}
\Delta u=|x|^{\mu}|v|^{q-1} v \quad \text { in } B_{R}, \\
\Delta v=|x|^{v}|u|^{p} \quad \text { in } B_{R}, \\
u=\frac{\partial u}{\partial v}=0 \quad \text { on } \partial B_{R},
\end{gathered}
$$

where $\mu, v \geq 0$, has at most one nontrivial radial solution $(u, v)$. Indeed, the arguments are the same with $s$ and $t$ in (2.1) replaced by

$$
s=\frac{2(q+1)+v+q \mu}{p q-1}, \quad t=\frac{2(p+1)+\mu+p v}{p q-1} .
$$

Now let $p>0, q \geq 1$ with $p q=1$. Assume that problem (3.10) has a nontrivial radial solution $(u, v)$. Then all nontrivial radial solutions are given by $\left(\theta^{q} u, \theta v\right)$, where $\theta>0$ is an arbitrary constant.

\section{Proof of Theorem 1.2}

(i) Let $(u, v) \in\left(C^{2}\left(\bar{B}_{R}\right)\right)^{2}$ be a solution of problem (1.1) such that $u \geq 0$ in $B_{R}$. We have $x \cdot \nu(x)=R$ for all $x \in \partial B_{R}$. Multiplying the first equation in (1.1) by $x \cdot \nabla v$ and integrating over $B_{R}$, we get

$$
\int_{B_{R}}(x \cdot \nabla v) \Delta u d x=\int_{B_{R}}(x \cdot \nabla v)|v|^{q-1} v d x .
$$

Integrating by parts, we obtain

$$
\int_{B_{R}}(x \cdot \nabla v)|v|^{q-1} v d x=-\frac{n}{q+1} \int_{B_{R}}|v|^{q+1} d x+\frac{R}{q+1} \int_{\partial B_{R}}|v|^{q+1} d \sigma .
$$


Similarly we get

$$
\int_{B_{R}}(x \cdot \nabla u) \Delta v d x=\int_{B_{R}}(x \cdot \nabla u) u^{p} d x=-\frac{n}{p+1} \int_{B_{R}} u^{p+1} d x .
$$

Now we have

$$
\int_{B_{R}}((x \cdot \nabla v) \Delta u+(x \cdot \nabla u) \Delta v) d x=(n-2) \int_{B_{R}} \nabla u \cdot \nabla v d x .
$$

Then we deduce that

$$
\frac{R}{q+1} \int_{\partial B_{R}}|v|^{q+1} d \sigma=\frac{n}{q+1} \int_{B_{R}}|v|^{q+1} d x+\frac{n}{p+1} \int_{B_{R}} u^{p+1} d x+(n-2) \int_{B_{R}} \nabla u \cdot \nabla v d x
$$

Since

$$
\begin{aligned}
& \int_{B_{R}} \nabla u \cdot \nabla v d x=-\int_{B_{R}} v \Delta u d x=-\int_{B_{R}}|v|^{q+1} d x, \\
& \int_{B_{R}} \nabla u \cdot \nabla v d x=-\int_{B_{R}} u \Delta v d x=-\int_{B_{R}} u^{p+1} d x,
\end{aligned}
$$

we can write

$$
\frac{R}{q+1} \int_{\partial B_{R}}|v|^{q+1} d \sigma=n\left(\frac{1}{p+1}+\frac{1}{q+1}-\frac{n-2}{n}\right) \int_{B_{R}}|v|^{q+1} d x .
$$

Using (1.4) we deduce that $v=0$ on $\partial B_{R}$. The maximum principle implies that $v \leq 0$ in $B_{R}$. Therefore $\Delta u \leq 0$ in $B_{R}$. The Hopf boundary point lemma implies that $u=0$ in $B_{R}$ and (i) is proved.

(ii) follows from (i) and Lemma 2.1.

Remark 4.1. Clearly Theorem 1.2(i) can be extended to more general domains and more general nonlinearities as in $[2,11,12]$ and Theorem 1.2 (ii) can be extended to more general nonlinearities.

\section{Proof of Theorem 1.3}

We will use a two-dimensional shooting argument for the ordinary differential equations associated to radial solutions of $(1.1)[3,5,7,15,16]$. We consider the one-dimensional (singular if $n \geq 2$ ) initial value problem (2.2) where $\alpha>0, \beta>0$.

We will need a series of lemmas. We begin with a standard local existence and uniqueness result.

Lemma 5.1. For any $\alpha>0, \beta>0$ there exists $T=T(\alpha, \beta)>0$ such that problem (2.2) on $[0, T]$ has a unique solution $(u, v) \in\left(C^{2}[0, T]\right)^{2}$. 
1514 Existence and uniqueness for an elliptic system

Proof. Let $\alpha, \beta>0$ be given. Choose $T=T(\alpha, \beta)>0$ such that

$$
T=\min \left(\left(\frac{n \alpha}{\beta^{q}}\right)^{1 / 2},\left(\frac{n \beta}{\alpha^{p}}\right)^{1 / 2}\right),
$$

and consider the set of functions

$$
Z=\left\{(u, v) \in(C[0, T])^{2} ; \frac{\alpha}{2} \leq u(r) \leq \alpha,-\beta \leq v(r) \leq-\frac{\beta}{2} \text { for } 0 \leq r \leq T\right\} .
$$

Clearly $Z$ is a bounded closed convex subset of the Banach space $(C[0, T])^{2}$ endowed with the norm $\|(u, v)\|=\max \left(\|u\|_{\infty},\|v\|_{\infty}\right)$. Define

$$
L(u, v)(r)=\left(\alpha+\int_{0}^{r} G_{n}(r, s)|v(s)|^{q-1} v(s) d s,-\beta+\int_{0}^{r} G_{n}(r, s)|u(s)|^{p} d s\right)
$$

for $r \in[0, T]$ and $(u, v) \in(C[0, T])^{2}$, where $G_{n}$ is defined in (3.8). It is easily verified that $L$ is a compact operator mapping $Z$ into itself, and so there exists $(u, v) \in Z$ such that $(u, v)=L(u, v)$ by the Schauder fixed point theorem. Clearly $(u, v) \in\left(C^{2}[0, T]\right)^{2}$ and $(u, v)$ is a solution of $(2.2)$ on $[0, T]$. Since the right-hand side in (2.2) is Lipschitz continuous in $(u, v) \in[\alpha / 2, \alpha] \times[-\beta,-\beta / 2]$, the uniqueness follows.

Remark 5.2. Notice that $u(r)>0$ and $v(r)<0$ for $r \in[0, T]$. Then direct integration of the system (2.2) implies that $u^{\prime}<0$ and $v^{\prime}>0$ in $(0, T]$.

In view of Lemma 5.1, for any $\alpha, \beta>0$ problem (2.2) has a unique local solution: let $\left[0, R_{\alpha, \beta}\right)$ denote the maximum interval of existence of that solution $\left(R_{\alpha, \beta}=+\infty\right.$ possibly). If $0<p<1$, the uniqueness of the solution could fail at any point $r$ where $u(r)=0$. In this case, $R_{\alpha, \beta}$ could also depend on the particular solution itself. Define

$$
P_{\alpha, \beta}=\left\{s \in\left(0, R_{\alpha, \beta}\right) ; u(\alpha, \beta, r) u^{\prime}(\alpha, \beta, r)<0 \forall r \in(0, s]\right\},
$$

where $(u(\alpha, \beta, \cdot), v(\alpha, \beta, \cdot))$ is a solution of $(2.2)$ in $\left[0, R_{\alpha, \beta}\right) . P_{\alpha, \beta} \neq \varnothing$ by Remark 5.2. Set

$$
r_{\alpha, \beta}=\sup P_{\alpha, \beta}
$$

Notice that the solution is unique on $\left[0, r_{\alpha, \beta}\right]$, so $r_{\alpha, \beta}$ depends only on $\alpha, \beta$.

Lemma 5.3. $u^{\prime}(\alpha, \beta, r)<0$ for $r \in\left(0, r_{\alpha, \beta}\right)$ and $v^{\prime}(\alpha, \beta, r)>0$ for $r \in\left(0, R_{\alpha, \beta}\right)$.

Proof. The first assertion follows from the definition of $r_{\alpha, \beta}$. Since $u(\alpha, \beta, r)>0$ for $r \in$ $\left[0, r_{\alpha, \beta}\right)$, integrating the second equation in (2.2) from 0 to $r \in\left(0, R_{\alpha, \beta}\right)$ we obtain $v^{\prime}(\alpha, \beta$, $r)>0$ for $r \in\left(0, R_{\alpha, \beta}\right)$.

LeMma 5.4. For any $\alpha, \beta>0, r_{\alpha, \beta}<\infty$.

Proof. Assume that $r_{\alpha, \beta}=\infty$. We easily get a contradiction when $n=1$ or 2 . Now if $n \geq 3$, we set $z=-v$. By Lemma $2.2, z>0$ on $[0, \infty)$ and we have

$$
\begin{array}{ll}
-\Delta u=z^{q}, & r>0, \\
-\Delta z=u^{p}, & r>0 .
\end{array}
$$


Since $p, q$ satisfy (1.5), we obtain a contradiction with the help of the nonexistence results established in $[9,10,13,14]$.

Lemma 5.5. For any $a \in\left[T(\alpha, \beta), r_{\alpha, \beta}\right)$, there exists $b=b(\alpha, \beta, a)>0$ such that the maximal extension of $(u, v)$ includes the interval $[0, a+b]$. Moreover,

$$
b(\alpha, \beta, a)=\frac{m(\alpha, \beta)}{a+\sqrt{a^{2}+m(\alpha, \beta)}},
$$

where

$$
m(\alpha, \beta)=\min \left(\frac{n \beta}{2^{p-1} \alpha^{p}}, \frac{n \alpha}{2^{q-1} d^{q}\left(\max \left(\beta, \alpha^{(p+1) /(q+1)}\right)\right)^{q}}\right),
$$

with d given in Lemma 2.3.

Proof. Lemma 5.5 is essentially a local existence result, with initial data $u(a), v(a), u^{\prime}(a)$, $v^{\prime}(a)$ at $r=a$. Let

$$
W=\left\{(u, v) \in(C[a, a+b])^{2} ;|u(r)-u(a)| \leq \alpha, 0 \leq v(r)-v(a) \leq \beta \text { for } a \leq r \leq a+b\right\},
$$

where $b=b(\alpha, \beta, a)$ is given in the lemma. $W$ is a bounded closed convex subset of the $\mathrm{Ba}$ nach space $(C[a, a+b])^{2}$ equipped with the norm $\|(u, v)\|=\max \left(\|u\|_{\infty},\|v\|_{\infty}\right)$. Consider the mapping $S(u, v)=\left(S_{1}(u, v), S_{2}(u, v)\right)$ on $(C[a, a+b])^{2}$ given by

$$
\begin{gathered}
S_{1}(u, v)(r)=u(a)+\int_{a}^{r} \frac{d t}{t^{n-1}} \int_{0}^{t} s^{n-1}|v(s)|^{q-1} v(s) d s, \\
S_{2}(u, v)(r)=v(a)+\int_{a}^{r} \frac{d t}{t^{n-1}} \int_{0}^{t} s^{n-1}|u(s)|^{p} d s
\end{gathered}
$$

for $a \leq r \leq a+b$, where we also denote by $u, v$ the unique solution of $(2.2)$ on $[0, a]$. Let $(u, v) \in W$. Using Lemma 5.3, we have

$$
|u(s)| \leq u(a)+\alpha \leq 2 \alpha, \quad s \in[a, a+b] .
$$

Therefore we get

$$
0 \leq S_{2}(u, v)(r)-v(a) \leq 2^{p-1} \alpha^{p} \frac{r^{2}-a^{2}}{n} \leq \beta, \quad r \in[a, a+b] .
$$

By Lemma 2.3 we have

$$
|v(s)| \leq|v(a)|+\beta \leq 2 d \max \left(\beta, \alpha^{(p+1) /(q+1)}\right), \quad s \in[a, a+b] .
$$

Therefore for $a \leq r \leq a+b$, we obtain

$$
\left|S_{1}(u, v)(r)-u(a)\right| \leq 2^{q-1} d^{q}\left(\max \left(\beta, \alpha^{(p+1) /(q+1)}\right)\right)^{q} \frac{r^{2}-a^{2}}{n} \leq \alpha .
$$


1516 Existence and uniqueness for an elliptic system

We have thus proved that $S(W) \subset W$. Since $S$ is a compact operator, there exists $(u, v) \in W$ such that $(u, v)=S(u, v)$ by the Schauder fixed point theorem. Clearly $(u, v) \in$ $\left(C^{2}[a, a+b]\right)^{2}$ and $(u, v)$ is a solution of $(2.2)$ on $[a, a+b]$ which extends the solution $(u, v)$ on $[0, a]$.

LEMma 5.6. For any $\alpha, \beta>0$,

$$
R_{\alpha, \beta} \geq r_{\alpha, \beta}+\frac{m(\alpha, \beta)}{r_{\alpha, \beta}+\sqrt{r_{\alpha, \beta}^{2}+m(\alpha, \beta)}} .
$$

Proof. By Lemma 5.5, for any $a \in\left(T(\alpha, \beta), r_{\alpha, \beta}\right)$ we have

$$
R_{\alpha, \beta}>a+\frac{m(\alpha, \beta)}{a+\sqrt{a^{2}+m(\alpha, \beta)}} .
$$

The lemma follows by letting $a \rightarrow r_{\alpha, \beta}$.

Proposition 5.7. For any $\alpha>0$, there exists a unique $\beta>0$ such that $u\left(\alpha, \beta, r_{\alpha, \beta}\right)=u^{\prime}(\alpha, \beta$, $\left.r_{\alpha, \beta}\right)=0$.

Proof. We first prove the uniqueness. Let $\alpha>0$ be fixed. Suppose that there exist $\beta>$ $\gamma>0$ such that $u\left(\alpha, \beta, r_{\alpha, \beta}\right)=u^{\prime}\left(\alpha, \beta, r_{\alpha, \beta}\right)=u\left(\alpha, \gamma, r_{\alpha, \gamma}\right)=u^{\prime}\left(\alpha, \gamma, r_{\alpha, \gamma}\right)=0$. Using the same arguments as in the proof of (3.5) we obtain a contradiction.

Now we prove the existence. Suppose that there exists $\alpha>0$ such that for any $\beta>0$ $u\left(\alpha, \beta, r_{\alpha, \beta}\right)>0$ or $u^{\prime}\left(\alpha, \beta, r_{\alpha, \beta}\right)<0$. Define the sets

$$
\begin{aligned}
& B=\left\{\beta>0 ; u\left(\alpha, \beta, r_{\alpha, \beta}\right)=0, u^{\prime}\left(\alpha, \beta, r_{\alpha, \beta}\right)<0\right\} \\
& C=\left\{\beta>0 ; u\left(\alpha, \beta, r_{\alpha, \beta}\right)>0, u^{\prime}\left(\alpha, \beta, r_{\alpha, \beta}\right)=0\right\}
\end{aligned}
$$

The proof of the proposition is completed by using the next two lemmas which contradict the fact that

$$
(0,+\infty)=B \cup C
$$

Lemma 5.8. (i) Suppose $B \neq \varnothing$. Then there exists $m>0$ such that $m \leq \inf B$.

(ii) Suppose $C \neq \varnothing$. Then there exists $M>0$ such that $M \geq \sup C$.

LEMMA 5.9. $B$ and $C$ are open.

Proof of Lemma 5.8. We have

$$
\begin{array}{rr}
u(\alpha, \beta, r)=\alpha+\int_{0}^{r} G_{n}(r, s)|v(\alpha, \beta, s)|^{q-1} v(\alpha, \beta, s) d s, & 0 \leq r<R_{\alpha, \beta}, \\
v(\alpha, \beta, r)=-\beta+\int_{0}^{r} G_{n}(r, s)|u(\alpha, \beta, s)|^{p} d s, & 0 \leq r<R_{\alpha, \beta} .
\end{array}
$$


(i) Let $\beta \in B$. Assume first that $v(\alpha, \beta, \cdot)<0$ on $\left[0, r_{\alpha, \beta}\right)$. Then Lemma 5.3 and (5.19) imply

$$
r_{\alpha, \beta} \geq\left(\frac{2 n \alpha}{\beta^{q}}\right)^{1 / 2}
$$

Now, if there exists $s_{\alpha, \beta} \in\left[0, r_{\alpha, \beta}\right)$ such that $v\left(\alpha, \beta, s_{\alpha, \beta}\right)=0$, Lemma 5.3 implies that $-\beta \leq$ $v(\alpha, \beta, \cdot)<0$ in $\left[0, s_{\alpha, \beta}\right)$ and $v(\alpha, \beta, \cdot)>0$ in $\left(s_{\alpha, \beta}, r_{\alpha, \beta}\right]$. Then from (5.19) we get

$$
\begin{aligned}
\alpha & =-\int_{0}^{r_{\alpha, \beta}} G_{n}\left(r_{\alpha, \beta}, s\right)|v(\alpha, \beta, s)|^{q-1} v(\alpha, \beta, s) d s \\
& \leq \int_{0}^{s_{\alpha, \beta}} G_{n}\left(r_{\alpha, \beta}, s\right)|v(\alpha, \beta, s)|^{q} d s \leq \beta^{q} \int_{0}^{s_{\alpha, \beta}} G_{n}\left(r_{\alpha, \beta}, s\right) d s \leq \beta^{q} \frac{r_{\alpha, \beta}^{2}}{2 n},
\end{aligned}
$$

and (5.21) still holds.

Suppose that $\inf B=0$ and let $\left(\beta_{j}\right)$ be a sequence in $B$ decreasing to zero. Then $r_{\alpha, \beta_{j}} \rightarrow$ $+\infty$ by (5.21). Let $r>0$ be fixed. We can assume that $r_{\alpha, \beta_{j}}>r$ for all $j$. If $v\left(\alpha, \beta_{j}, s\right)<0$ for $s \in[0, r]$, we have

$$
u\left(\alpha, \beta_{j}, r\right)=\alpha-\int_{0}^{r} G_{n}(r, s)\left|v\left(\alpha, \beta_{j}, s\right)\right|^{q} d s \geq \alpha-\frac{r^{2} \beta_{j}^{q}}{2 n} .
$$

If $s_{\alpha, \beta_{j}}<r$, we have

$$
\begin{aligned}
u\left(\alpha, \beta_{j}, r\right) & =\alpha-\int_{0}^{s_{\alpha, \beta_{j}}} G_{n}(r, s)\left|v\left(\alpha, \beta_{j}, s\right)\right|^{q} d s+\int_{s_{\alpha, \beta_{j}}}^{r} G_{n}(r, s) v\left(\alpha, \beta_{j}, s\right)^{q} d s \\
& \geq \alpha-\int_{0}^{s_{\alpha, \beta_{j}}} G_{n}(r, s)\left|v\left(\alpha, \beta_{j}, s\right)\right|^{q} d s \\
& \geq \alpha-\beta_{j}^{q} \int_{0}^{s_{\alpha, \beta_{j}}} G_{n}(r, s) d s \geq \alpha-\frac{r^{2} \beta_{j}^{q}}{2 n} .
\end{aligned}
$$

Therefore using Lemma 5.3 we obtain

$$
u\left(\alpha, \beta_{j}, s\right) \geq \alpha-\frac{r^{2} \beta_{j}^{q}}{2 n} \quad \text { for } s \in[0, r],
$$

from which we deduce that

$$
u\left(\alpha, \beta_{j}, s\right) \geq \frac{\alpha}{2}
$$

for $s \in[0, r]$ and $j$ large. From (5.20) we get

$$
v\left(\alpha, \beta_{j}, r\right) \geq-\beta_{j}+\frac{r^{2} \alpha^{p}}{2^{p+1} n}
$$

for $j$ large. Thus if we choose $r$ such that

$$
-\beta_{j}+\frac{r^{2} \alpha^{p}}{2^{p+1} n} \geq 1
$$


1518 Existence and uniqueness for an elliptic system

using Lemma 5.3 we get

$$
v\left(\alpha, \beta_{j}, s\right) \geq 1
$$

for $r \leq s \leq r_{\alpha, \beta_{j}}$ and $j$ large. We also have

$$
-\beta_{j} \leq v\left(\alpha, \beta_{j}, s\right) \leq-\beta_{j}+\frac{r^{2} \alpha^{p}}{2 n}
$$

for $s \in[0, r]$. Therefore there exists $c>0$ such that

$$
\left|v\left(\alpha, \beta_{j}, s\right)\right| \leq c
$$

for $s \in[0, r]$ and all $j$. There exists $k>0$ such that

$$
\int_{r}^{r_{\alpha, \beta_{j}}} G_{n}\left(r_{\alpha, \beta_{j}}, s\right) d s \geq k r_{\alpha, \beta_{j}}^{2}
$$

for $j$ large. Now we write

$$
\begin{aligned}
\alpha= & -\int_{0}^{r_{\alpha, \beta_{j}}} G_{n}\left(r_{\alpha, \beta_{j}}, s\right)\left|v\left(\alpha, \beta_{j}, s\right)\right|^{q-1} v\left(\alpha, \beta_{j}, s\right) d s \\
= & -\int_{0}^{r} G_{n}\left(r_{\alpha, \beta_{j}}, s\right)\left|v\left(\alpha, \beta_{j}, s\right)\right|^{q-1} v\left(\alpha, \beta_{j}, s\right) d s \\
& -\int_{r}^{r_{\alpha, \beta_{j}}} G_{n}\left(r_{\alpha, \beta_{j}}, s\right) v\left(\alpha, \beta_{j}, s\right)^{q} d s \\
\leq & c^{q} \int_{0}^{r} G_{n}\left(r_{\alpha, \beta_{j}}, s\right) d s-\int_{r}^{r_{\alpha, \beta_{j}}} G_{n}\left(r_{\alpha, \beta_{j}}, s\right) d s \\
\leq & c^{q} r r_{\alpha, \beta_{j}}-k r_{\alpha, \beta_{j}}^{2}
\end{aligned}
$$

for $j$ large, where we have used the fact that $G_{n}\left(r_{\alpha, \beta_{j}}, s\right) \leq r_{\alpha, \beta_{j}}-s$ for $0 \leq s \leq r_{\alpha, \beta_{j}}$. Since the last term above tends to $-\infty$, we get a contradiction.

(ii) Let $\beta \in C$. We claim that $v\left(\alpha, \beta, r_{\alpha, \beta}\right)>0$. If not, by Lemma 5.3 we have $\Delta u(\alpha, \beta, \cdot)<$ 0 on $\left[0, r_{\alpha, \beta}\right)$ for some $\beta \in C$. Since $u^{\prime}(\alpha, \beta, 0)=0$, we obtain $u^{\prime}\left(\alpha, \beta, r_{\alpha, \beta}\right)<0$, a contradiction. Therefore (5.20) implies

$$
\beta<\int_{0}^{r_{\alpha, \beta}} G_{n}\left(r_{\alpha, \beta}, s\right) u(\alpha, \beta, s)^{p} d s
$$

for $\beta \in C$. Suppose that $\sup C=+\infty$ and let $\left(\beta_{j}\right)$ be a sequence in $C$ increasing to $+\infty$. Since $0<u\left(\alpha, \beta_{j}, r\right) \leq \alpha$ for $r \in\left[0, r_{\alpha, \beta_{j}}\right]$, (5.34) implies that $r_{\alpha, \beta_{j}} \rightarrow+\infty$ as $j \rightarrow+\infty$. Then we can assume that $r_{\alpha, \beta_{j}} \geq 1$ and that $\alpha^{p} \leq \beta_{j}$ for all $j$. From (5.20) we get

$$
-\beta_{j} \leq v\left(\alpha, \beta_{j}, r\right) \leq-\frac{2 n-1}{2 n} \beta_{j} \leq-\frac{\beta_{j}}{2} \quad \text { for } r \in[0,1],
$$

and using (5.19) we deduce that $u\left(\alpha, \beta_{j}, 1\right) \leq \alpha-\beta_{j}^{q} / n 2^{q+1}$. But then $u\left(\alpha, \beta_{j}, 1\right)<0$ for $j$ large and we reach a contradiction. 
Remark 5.10. The proof above shows that, when $\beta \in C$, there exists $s_{\alpha, \beta} \in\left(0, r_{\alpha, \beta}\right)$ such that $v(\alpha, \beta, \cdot)<0$ on $\left[0, s_{\alpha, \beta}\right)$ and $v(\alpha, \beta, \cdot)>0$ on $\left(s_{\alpha, \beta}, r_{\alpha, \beta}\right]$. When $\beta \in B, s_{\alpha, \beta}$ may not exist.

\section{Proof of Lemma 5.9}

Case $1(p \geq 1)$. Then the right-hand side of (2.2) is Lipschitz continuous. Let $\beta \in B$. We have $u\left(\alpha, \beta, r_{\alpha, \beta}\right)=0$ and $u^{\prime}\left(\alpha, \beta, r_{\alpha, \beta}\right)<0$. Therefore we can find $\varepsilon>0$ such that

$$
u\left(\alpha, \beta, r_{\alpha, \beta}+\varepsilon\right)<0, \quad u^{\prime}\left(\alpha, \beta, r_{\alpha, \beta}+\varepsilon\right)<0 .
$$

But then by continuous dependence on initial data, there exists $\eta>0$ such that

$$
u\left(\alpha, \gamma, r_{\alpha, \beta}+\varepsilon\right)<0, \quad u^{\prime}\left(\alpha, \gamma, r_{\alpha, \beta}+\varepsilon\right)<0
$$

for $|\gamma-\beta|<\eta$. The first inequality in (5.37) implies that there exists $x \in\left(0, r_{\alpha, \beta}+\varepsilon\right)$ such that $u(\alpha, \gamma, x)=0$ and $u(\alpha, \gamma, r)>0$ for $r \in[0, x) . \Delta v(\alpha, \gamma, r)>0$ for $r \in[0, x)$ and $\Delta v(\alpha, \gamma, r) \geq 0$ for $r \in\left[x, r_{\alpha, \beta}+\varepsilon\right]$. Then $v^{\prime}(\alpha, \gamma, r)>0$ for $r \in\left(0, r_{\alpha, \beta}+\varepsilon\right]$ and $v(\alpha, \gamma, \cdot)$ is increasing on $\left[0, r_{\alpha, \beta}+\varepsilon\right]$. We deduce that $\Delta u(\alpha, \gamma, \cdot)$ is increasing on $\left[0, r_{\alpha, \beta}+\varepsilon\right]$. If $\Delta u\left(\alpha, \gamma, r_{\alpha, \beta}+\varepsilon\right) \leq 0$, then $u^{\prime}(\alpha, \gamma, r)<0$ for $r \in\left(0, r_{\alpha, \beta}+\varepsilon\right]$. If $\Delta u\left(\alpha, \gamma, r_{\alpha, \beta}+\varepsilon\right)>0$, then there exists $s_{\alpha, \gamma} \in\left(0, r_{\alpha, \beta}+\varepsilon\right)$ such that $\Delta u(\alpha, \gamma, \cdot)<0$ in $\left[0, s_{\alpha, \gamma}\right)$ and $\Delta u(\alpha, \gamma, \cdot)>0$ in $\left(s_{\alpha, \gamma}, r_{\alpha, \beta}+\varepsilon\right]$. We deduce that $u^{\prime}(\alpha, \gamma, \cdot)$ is decreasing (resp., increasing) in $\left[0, s_{\alpha, \gamma}\right]$ (resp., $\left.\left[s_{\alpha, \gamma}, r_{\alpha, \beta}+\varepsilon\right]\right)$. Since $u^{\prime}(\alpha, \gamma, 0)=0$, the second inequality in (5.37) implies that $u^{\prime}(\alpha, \gamma, r)<0$ for $r \in\left(0, r_{\alpha, \beta}+\varepsilon\right]$. Therefore $x=r_{\alpha, \gamma}$ for $|\gamma-\beta|<\eta$ and $(\beta-\eta, \beta+\eta) \subset$ $B$. Thus $B$ is open. Now let $\beta \in C$. We have $u\left(\alpha, \beta, r_{\alpha, \beta}\right)>0$ and $u^{\prime}\left(\alpha, \beta, r_{\alpha, \beta}\right)=0$. By Remark 5.10, we have $v\left(\alpha, \beta, r_{\alpha, \beta}\right)>0$, hence $\Delta u\left(\alpha, \beta, r_{\alpha, \beta}\right)=u^{\prime \prime}\left(\alpha, \beta, r_{\alpha, \beta}\right)>0$. Therefore we can find $\varepsilon>0$ such that

$$
u(\alpha, \beta, r)>0, \quad r \in\left[0, r_{\alpha, \beta}+\varepsilon\right], \quad u^{\prime}\left(\alpha, \beta, r_{\alpha, \beta}+\varepsilon\right)>0 .
$$

Then by continuous dependence on initial data, there exists $\eta>0$ such that

$$
u(\alpha, \gamma, r)>0, \quad r \in\left[0, r_{\alpha, \beta}+\varepsilon\right], \quad u^{\prime}\left(\alpha, \gamma, r_{\alpha, \beta}+\varepsilon\right)>0
$$

for $|\gamma-\beta|<\eta$. The second inequality in (5.39) implies that there exists $x \in\left(0, r_{\alpha, \beta}+\varepsilon\right)$ such that $u^{\prime}(\alpha, \gamma, x)=0$ and $u^{\prime}(\alpha, \gamma, r)<0$ for $r \in(0, x)$. Therefore $x=r_{\alpha, \gamma}$ for $|\gamma-\beta|<\eta$ and $(\beta-\eta, \beta+\eta) \subset C$. Thus $C$ is open.

Case $2(0<p<1)$. We first show that $C$ is open. Indeed let $\beta \in C$. Since $u(\alpha, \beta, r)>0$ for $r \in\left[0, r_{\alpha, \beta}\right]$, the system (2.2) is Lipschitz continuous in $u$ and $v$ when $u$ is in a neighborhood of the interval $\left[u\left(\alpha, \beta, r_{\alpha, \beta}\right), \alpha\right]$ in $(0, \infty)$, and the solution $u(\alpha, \beta, \cdot), v(\alpha, \beta, \cdot)$ can be uniquely extended to $\left[0, r_{\alpha, \beta}+t\right]$ for some $t>0$, with $u(\alpha, \beta, r)>0$ for $r \in\left[0, r_{\alpha, \beta}+t\right]$. Then we can argue as in Case 1. Now we show that $B$ is open. As in [15], this case is much more difficult. We begin with the following two steps. Let $\beta \in B$.

Step 1. There exists $c>0$ and $\eta>0$ such that when $|\beta-\gamma|<\eta$, the solutions $u(\alpha, \gamma, \cdot)$, $v(\alpha, \gamma, \cdot)$, and $u(\alpha, \beta, \cdot), v(\alpha, \beta, \cdot)$ are defined on $\left[0, r_{\alpha, \beta}+c\right]$. 
By Lemma 5.6, $u(\alpha, \beta, \cdot), v(\alpha, \beta, \cdot)$ can be extended to the interval $\left[0, r_{\alpha, \beta}+b\left(\alpha, \beta, r_{\alpha, \beta}\right)\right)$ where

$$
b\left(\alpha, \beta, r_{\alpha, \beta}\right)=\frac{m(\alpha, \beta)}{r_{\alpha, \beta}+\sqrt{r_{\alpha, \beta}^{2}+m(\alpha, \beta)}} .
$$

Fix $\omega \in\left(0, r_{\alpha, \beta}-T(\alpha, \beta)\right)$ and $\mu=r_{\alpha, \beta}-\omega$. Then $T(\alpha, \beta)<\mu<r_{\alpha, \beta}$ and by Lemma 5.3

$$
0<u(\alpha, \beta, \mu) \leq u(\alpha, \beta, r) \leq \alpha, \quad 0 \leq r \leq \mu .
$$

Since the system (2.2) is Lipschitz continuous in $u$ and $v$ when $u$ is in a neighborhood of the interval $[u(\alpha, \beta, \mu), \alpha]$ in $(0, \infty)$, the continuous dependence on initial data implies that there exists $\eta>0$ such that when $|\gamma-\beta|<\eta$ the solution $u(\alpha, \gamma, \cdot), v(\alpha, \gamma, \cdot)$ is defined on $[0, \mu]$ and $u(\alpha, \gamma, r)>0$ for $r \in[0, \mu], u^{\prime}(\alpha, \gamma, r)<0$ for $r \in(0, \mu]$, hence $r_{\alpha, \gamma}>\mu$. By taking $\eta$ smaller if necessary, we can assume that $T(\alpha, \gamma)<\mu$, hence $T(\alpha, \gamma)<\mu<r_{\alpha, \gamma}$. By Lemma 5.5 we can extend $u(\alpha, \gamma, \cdot), v(\alpha, \gamma, \cdot)$ to $[0, \mu+b(\alpha, \gamma, \mu)]$. By taking $\eta$ smaller if necessary, we can assume that

$$
b(\alpha, \gamma, \mu)>\frac{b(\alpha, \beta, \mu)}{2}>\frac{b\left(\alpha, \beta, r_{\alpha, \beta}\right)}{2}=2 c .
$$

Thus if we choose $\omega$ to satisfy also $\omega \leq c$, we get

$$
\mu+b(\alpha, \gamma, \mu)=r_{\alpha, \beta}-\omega+b(\alpha, \gamma, \mu) \geq r_{\alpha, \beta}+c
$$

Thus $u(\alpha, \gamma, \cdot), v(\alpha, \gamma, \cdot)$ extend to the interval $\left[0, r_{\alpha, \beta}+c\right]$ and $c<b\left(\alpha, \beta, r_{\alpha, \beta}\right)$ so that $u(\alpha, \beta, \cdot), v(\alpha, \beta, \cdot)$ also exist on $\left[0, r_{\alpha, \beta}+c\right]$.

Step 2. We claim that there exist $\varepsilon \in(0, c)$ and $\delta \in(0, \eta)$ such that

$$
\left|u^{\prime}(\alpha, \gamma, r)-u^{\prime}\left(\alpha, \beta, r_{\alpha, \beta}\right)\right| \leq \frac{1}{2}\left|u^{\prime}\left(\alpha, \beta, r_{\alpha, \beta}\right)\right|
$$

(recall that $u^{\prime}\left(\alpha, \beta, r_{\alpha, \beta}\right)<0$ ) when $|\gamma-\beta|<\delta$ and $\left|r-r_{\alpha, \beta}\right| \leq \varepsilon$. Let $\varepsilon \in(0, c),|\gamma-\beta|<\eta$, and $r \in\left[r_{\alpha, \beta}-\varepsilon, r_{\alpha, \beta}+\varepsilon\right]$. By Step 1 and integration of (2.2) we have

$$
\begin{aligned}
& u^{\prime}(\alpha, \gamma, r)-u^{\prime}\left(\alpha, \beta, r_{\alpha, \beta}\right) \\
&= u^{\prime}(\alpha, \gamma, r)-u^{\prime}(\alpha, \beta, r)+u^{\prime}(\alpha, \beta, r)-u^{\prime}\left(\alpha, \beta, r_{\alpha, \beta}\right) \\
&=\left(u^{\prime}\left(\alpha, \gamma, r_{\alpha, \beta}-\varepsilon\right)-u^{\prime}\left(\alpha, \beta, r_{\alpha, \beta}-\varepsilon\right)\right) \frac{\left(r_{\alpha, \beta}-\varepsilon\right)^{n-1}}{r^{n-1}} \\
&+\int_{r_{\alpha, \beta}-\varepsilon}^{r} \frac{s^{n-1}}{r^{n-1}}\left(|v(\alpha, \gamma, s)|^{q-1} v(\alpha, \gamma, s)-|v(\alpha, \beta, s)|^{q-1} v(\alpha, \beta, s)\right) d s \\
&+u^{\prime}\left(\alpha, \beta, r_{\alpha, \beta}\right)\left(\frac{r_{\alpha, \beta}^{n-1}}{r^{n-1}}-1\right)+\int_{r_{\alpha, \beta}}^{r} \frac{s^{n-1}}{r^{n-1}}|v(\alpha, \beta, s)|^{q-1} v(\alpha, \beta, s) d s .
\end{aligned}
$$


We deduce that

$$
\begin{aligned}
& \left|u^{\prime}(\alpha, \gamma, r)-u^{\prime}\left(\alpha, \beta, r_{\alpha, \beta}\right)\right| \\
& \leq\left|u^{\prime}\left(\alpha, \gamma, r_{\alpha, \beta}-\varepsilon\right)-u^{\prime}\left(\alpha, \beta, r_{\alpha, \beta}-\varepsilon\right)\right|+\left|u^{\prime}\left(\alpha, \beta, r_{\alpha, \beta}\right)\right|\left|\frac{r_{\alpha, \beta}^{n-1}}{r^{n-1}}-1\right| \\
& \quad+\int_{r_{\alpha, \beta}-\varepsilon}^{r} \frac{s^{n-1}}{r^{n-1}}|v(\alpha, \gamma, s)|^{q} d s+\int_{r_{\alpha, \beta}-\varepsilon}^{r_{\alpha, \beta}} \frac{s^{n-1}}{r^{n-1}}|v(\alpha, \beta, s)|^{q} d s .
\end{aligned}
$$

The proof of Lemma 5.5 gives the following estimate for $|\gamma-\beta|<\eta$ :

$$
|v(\alpha, \gamma, r)| \leq 2 d \max \left(\gamma, \alpha^{(p+1) /(q+1)}\right), \quad r_{\alpha, \beta}-\varepsilon \leq r \leq r_{\alpha, \beta}+\varepsilon .
$$

By making $\varepsilon$ smaller if necessary we have

$$
\begin{gathered}
\int_{r_{\alpha, \beta}-\varepsilon}^{r} \frac{s^{n-1}}{r^{n-1}}|v(\alpha, \gamma, s)|^{q} d s+\int_{r_{\alpha, \beta}-\varepsilon}^{r_{\alpha, \beta}} \frac{s^{n-1}}{r^{n-1}}|v(\alpha, \beta, s)|^{q} d s \leq \frac{1}{4}\left|u^{\prime}\left(\alpha, \beta, r_{\alpha, \beta}\right)\right|, \\
\qquad\left|\frac{r_{\alpha, \beta}^{n-1}}{r^{n-1}}-1\right| \leq \frac{1}{8}
\end{gathered}
$$

for $r_{\alpha, \beta}-\varepsilon \leq r \leq r_{\alpha, \beta}+\varepsilon$. Then from (5.46) we obtain

$$
\left|u^{\prime}(\alpha, \gamma, r)-u^{\prime}\left(\alpha, \beta, r_{\alpha, \beta}\right)\right| \leq\left|u^{\prime}\left(\alpha, \gamma, r_{\alpha, \beta}-\varepsilon\right)-u^{\prime}\left(\alpha, \beta, r_{\alpha, \beta}-\varepsilon\right)\right|+\frac{3}{8}\left|u^{\prime}\left(\alpha, \beta, r_{\alpha, \beta}\right)\right|
$$

for $|\gamma-\beta|<\eta$ and $\left|r-r_{\alpha, \beta}\right| \leq \varepsilon$. Now let $\varepsilon$ be fixed. By continuous dependence on initial data and the fact that $u(\alpha, \beta, r)>u\left(\alpha, \beta, r_{\alpha, \beta}-\varepsilon\right)$ for $r \in\left[0, r_{\alpha, \beta}-\varepsilon\right)$, we can choose $\delta \in$ $(0, \eta)$ such that

$$
\left|u^{\prime}\left(\alpha, \gamma, r_{\alpha, \beta}-\varepsilon\right)-u^{\prime}\left(\alpha, \beta, r_{\alpha, \beta}-\varepsilon\right)\right| \leq \frac{1}{8}\left|u^{\prime}\left(\alpha, \beta, r_{\alpha, \beta}\right)\right|
$$

for $|\gamma-\beta|<\delta$ and our claim follows.

Now assume that $B$ is not open. Equation (5.18) implies that there exist $\beta \in B$ and a sequence $\left(\beta_{j}\right)$ in $C$ such that $\beta_{j} \rightarrow \beta$ and $r_{\alpha, \beta_{j}} \rightarrow T \in[0, \infty]$. Assume first that $T>r_{\alpha, \beta}$. Then we can assume that there exists $c^{\prime} \in(0, c)$ such that $r_{\alpha, \beta_{j}} \geq r_{\alpha, \beta}+c^{\prime}$ for all $j$. We can also assume that $\varepsilon$ in Step 2 is such that $0<\varepsilon<c^{\prime}$. Since $u\left(\alpha, \beta, r_{\alpha, \beta}\right)=0$ and $u^{\prime}\left(\alpha, \beta, r_{\alpha, \beta}\right)<$ 0 , there exists $0<\varepsilon^{\prime} \leq \varepsilon$ such that

$$
0<u\left(\alpha, \beta, r_{\alpha, \beta}-\varepsilon^{\prime}\right)<\frac{1}{4}\left|u^{\prime}\left(\alpha, \beta, r_{\alpha, \beta}\right)\right| \varepsilon .
$$

By continuous dependence on initial data, there exists $\delta^{\prime} \in(0, \delta)$ such that

$$
u\left(\alpha, \gamma, r_{\alpha, \beta}-\varepsilon^{\prime}\right)<2 u\left(\alpha, \beta, r_{\alpha, \beta}-\varepsilon^{\prime}\right)
$$


1522 Existence and uniqueness for an elliptic system

when $|\gamma-\beta|<\delta^{\prime}$. Now let $j_{0}$ be such that $\left|\beta_{j}-\beta\right|<\delta^{\prime}$ for $j \geq j_{0}$. By Step 2 , for $\mid r-$ $r_{\alpha, \beta} \mid \leq \varepsilon$ and $j \geq j_{0}$ we have

$$
\left|u^{\prime}\left(\alpha, \beta_{j}, r\right)\right|=\left|u^{\prime}\left(\alpha, \beta, r_{\alpha, \beta}\right)\right|+u^{\prime}\left(\alpha, \beta, r_{\alpha, \beta}\right)-u^{\prime}\left(\alpha, \beta_{j}, r\right) \geq \frac{1}{2}\left|u^{\prime}\left(\alpha, \beta, r_{\alpha, \beta}\right)\right| .
$$

Therefore for $j \geq j_{0}$,

$$
\begin{aligned}
u\left(\alpha, \beta_{j}, r_{\alpha, \beta}+\varepsilon\right) & \leq u\left(\alpha, \beta_{j}, r_{\alpha, \beta}-\varepsilon^{\prime}\right)-\min _{\left|r-r_{\alpha, \beta}\right| \leq \varepsilon}\left|u^{\prime}\left(\alpha, \beta_{j}, r\right)\right|\left(\varepsilon+\varepsilon^{\prime}\right) \\
& <2 u\left(\alpha, \beta, r_{\alpha, \beta}-\varepsilon^{\prime}\right)-\frac{1}{2}\left|u^{\prime}\left(\alpha, \beta, r_{\alpha, \beta}\right)\right| \varepsilon<0
\end{aligned}
$$

Then we obtain a contradiction since $\beta_{j} \in C$. Now assume that $T \leq r_{\alpha, \beta}$. By Step 2 we have

$$
\left|u^{\prime}\left(\alpha, \beta_{j}, r_{\alpha, \beta_{j}}\right)-u^{\prime}\left(\alpha, \beta, r_{\alpha, \beta}\right)\right|=\left|u^{\prime}\left(\alpha, \beta, r_{\alpha, \beta}\right)\right| \leq \frac{1}{2}\left|u^{\prime}\left(\alpha, \beta, r_{\alpha, \beta}\right)\right|
$$

for $j \geq j_{0}$ and we get a contradiction.

Now we can complete the proof of Theorem 1.3.

(i) Let $\alpha>0$ be fixed. By Proposition 5.7, there exists a unique $\beta>0$ such that $u(\alpha, \beta$, $\left.r_{\alpha, \beta}\right)=u^{\prime}\left(\alpha, \beta, r_{\alpha, \beta}\right)=0$. With $s$ and $t$ defined in (2.1), we set

$$
w(r)=\left(\frac{r_{\alpha, \beta}}{R}\right)^{s} u\left(\alpha, \beta, \frac{r_{\alpha, \beta}}{R} r\right), \quad z(r)=\left(\frac{r_{\alpha, \beta}}{R}\right)^{t} v\left(\alpha, \beta, \frac{r_{\alpha, \beta}}{R} r\right), \quad 0 \leq r \leq R .
$$

Then $(w, z)$ is a nontrivial radial solution of problem (1.1).

(ii) follows from Proposition 5.7.

\section{Acknowledgment}

The author would like to thank the referees for the useful comments and suggestions.

\section{References}

[1] T. Boggio, Sulle funzioni di Green di ordine $m$, Rend. Circ. Mat. Palermo 20 (1905), 97-135 (Italian).

[2] R. Dalmasso, Problème de Dirichlet homogène pour une équation biharmonique semi-linéaire dans une boule [A homogeneous Dirichlet problem for a semilinear biharmonic equation in a ball], Bull. Sci. Math. 114 (1990), no. 2, 123-137 (French).

[3] Uniqueness of positive solutions of nonlinear second order systems, Rev. Mat. Iberoamericana 11 (1995), no. 2, 247-267.

[4] Uniqueness theorems for some fourth-order elliptic equations, Proc. Amer. Math. Soc. 123 (1995), no. 4, 1177-1183.

[5] Uniqueness of positive solutions for some nonlinear fourth-order equations, J. Math. Anal. Appl. 201 (1996), no. 1, 152-168.

[6] Existence and uniqueness results for polyharmonic equations, Nonlinear Anal. Ser. A: Theory Methods 36 (1999), no. 1, 131-137.

[7] Existence and uniqueness of positive radial solutions for the Lane-Emden system, Nonlinear Anal. 57 (2004), no. 3, 341-348. 
[8] H.-C. Grunau, The Dirichlet problem for some semilinear elliptic differential equations of arbitrary order, Analysis 11 (1991), no. 1, 83-90.

[9] E. Mitidieri, A Rellich type identity and applications, Comm. Partial Differential Equations 18 (1993), no. 1-2, 125-151.

[10] Non-existence of positive solutions of semilinear elliptic systems in $\mathbb{R}^{n}$, Differential Integral Equations 9 (1996), 456-479.

[11] P. Oswald, On a priori estimates for positive solutions of a semilinear biharmonic equation in a ball, Comment. Math. Univ. Carolin. 26 (1985), no. 3, 565-577.

[12] P. Pucci and J. Serrin, A general variational identity, Indiana Univ. Math. J. 35 (1986), no. 3, 681-703.

[13] J. Serrin and H. Zou, Non-existence of positive solutions of semilinear elliptic systems, A Tribute to Ilya Bakelman (College Station, Tex, 1993), Discourses Math. Appl., vol. 3, Texas A \& M University, Texas, 1994, pp. 55-68.

[14] - Non-existence of positive solutions of the Lane-Emden systems, Differential Integral Equations 9 (1996), no. 4, 635-653.

[15] Existence of positive entire solutions of elliptic Hamiltonian systems, Comm. Partial Differential Equations 23 (1998), no. 3-4, 577-599.

[16] Existence of positive solutions of the Lane-Emden system, Atti Sem. Mat. Fis. Univ. Modena 46 (1998), no. suppl., 369-380.

Robert Dalmasso: Equipe EDP, Laboratoire LMC-IMAG, Tour IRMA, BP 53, 38041 Grenoble Cedex 9, France

E-mail address: robert.dalmasso@imag.fr 


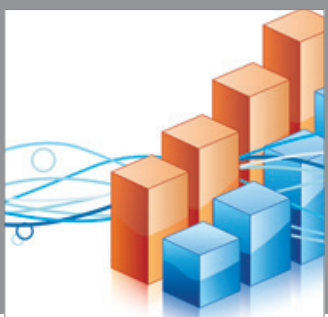

Advances in

Operations Research

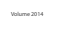

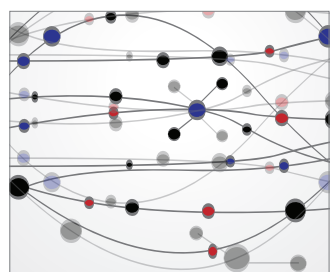

\section{The Scientific} World Journal
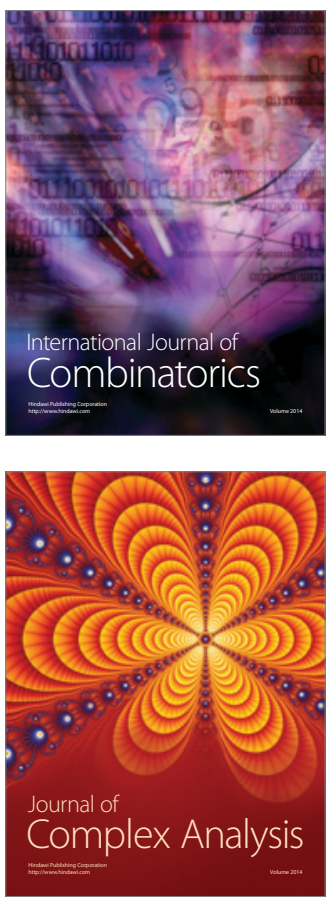

International Journal of

Mathematics and

Mathematical

Sciences
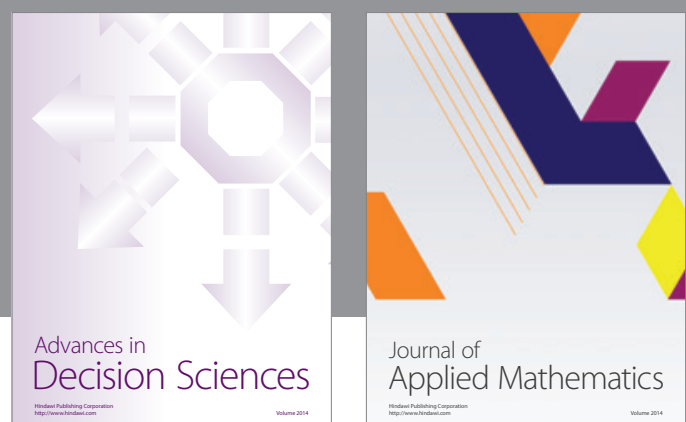

Journal of

Applied Mathematics
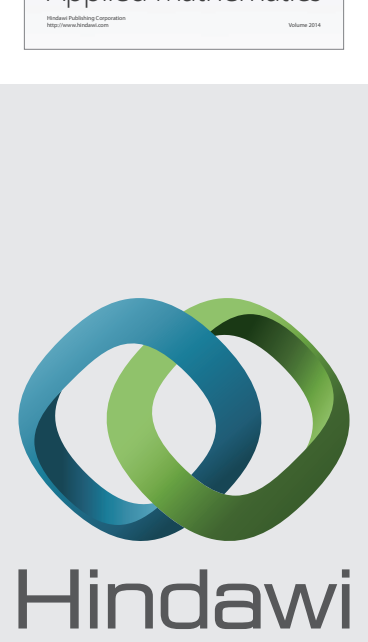

Submit your manuscripts at http://www.hindawi.com
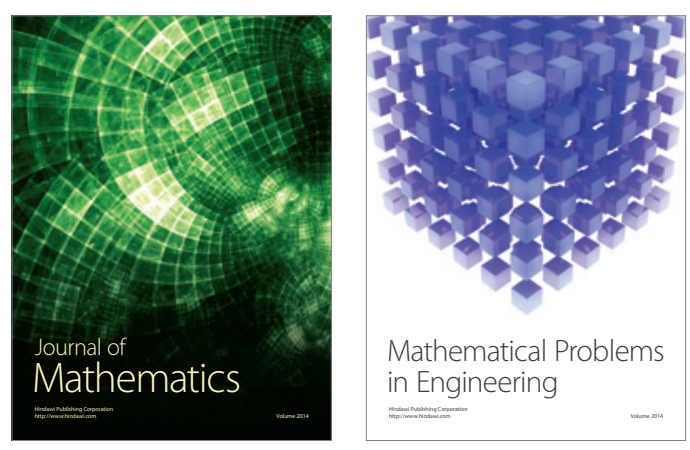

Mathematical Problems in Engineering
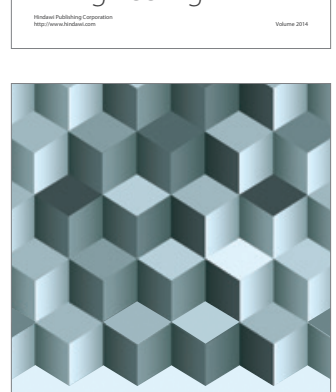

Journal of

Function Spaces
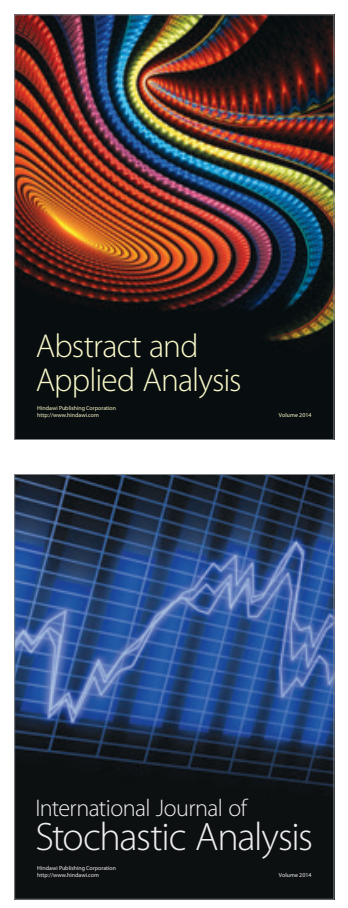

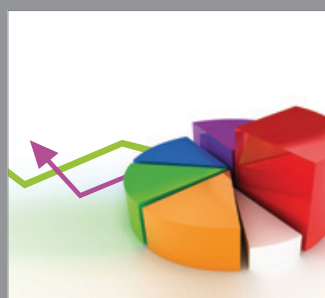

ournal of

Probability and Statistics

Promensencen
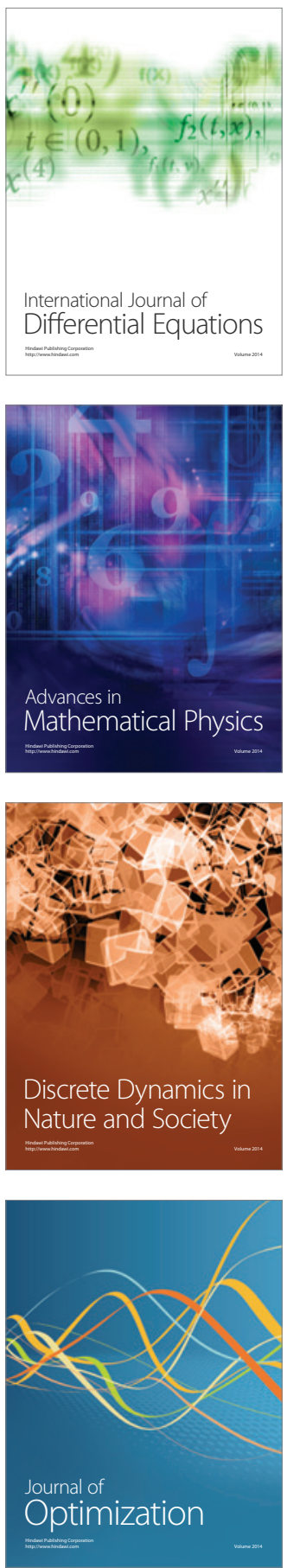